\title{
PEDIATRIC CHRONIC VIRAL HEPATITIS - THERAPEUTIC CONSIDERATIONS
}

\author{
Irina Dijmarescu, Daniela Pacurar, Dumitru Oraseanu \\ “Grigore Alexandrescu” Emergency Children's Hospital, Bucharest
}

\begin{abstract}
Chronic viral hepatitis is a public health issue both worldwide and in Romania, where the prevalence of the disease is still high. We present the available therapies used for chronic viral hepatitis B treatment in children, worldwide and in Romania, indications and contraindications of treatment, benefits and disadvantages for each class. The present paper reviews only the treatment options and not profilactic measures for pediatric chronic viral hepatitis B. Currently, in Romania, standard Interferon is the treatment of choice. Entecavir is the only of the nucleotidic/nucleosidic analogues which is approved for children use, but not in those weighting less than $32.6 \mathrm{~kg}$. Tenofovir, Adefovir and Lamivudine are not approved for pediatric chronic viral hepatitis B. Considering the fact that the disease progression is longer when the infection occurs earlier in life, efficient treatment is important for children with chronic viral hepatitis B. The availability of more treatment options provides a chance for patients who are nonresponsive to first line therapies.
\end{abstract}

Keywords: chronic viral hepatitis B, children, treatment

\section{INTRODUCTION}

Chronic viral hepatitis is one of the most common infectious diseases worldwide. Global prevalence is estimated at 350-400,000 million people, with the highest rate in sub-Saharian Africa and Eastern Asia (5-10\% of the adult population chronically infected). In Central and Eastern Europe and in Romania the reported prevalence is $2-5 \%$, while in North America and Western Europe it is below 1\%. (1) Forty five percent of the global population lives in hepatitis B highly endemic regions, while another $40 \%$ are located in intermediary prevalence regions. (2) One third of the world's population has been infected with hepatitis B virus and $5 \%$ are chronically infected, rarely during childhood (0-4\%). Approximately $25 \%$ of the chronically infected population will develop complications of the disease (cirrhosis and hepatocellular carcinoma) and about 780,000 people die annually from these complications. (3-5)

Chronic viral hepatitis is a public health issue, generating high morbidity and mortality, especially in developing countries, with insufficient therapeu- tic resources. Costs for prevention are lower than the costs for disease and complications treatment, both financially and regarding morbidity and mortality. In order to improve treatment results, new molecules have been developed, with better results, easier administration and fewer side effects. The present paper is a review of both well recognized treatment options and newer, recent methods. We do not present profilactic measures for chronic viral hepatitis B.

\section{ANTIVIRAL TREATMENT}

The aim of the antiviral treatment is to improve survival and to enhance the quality of life for patients, by reducing the risk of developing progressive liver disease, hepatocellular carcinoma or cirrhosis. The goal is stopping/inhibiting viral replication in order to reduce infectivity, stop disease progression and decrease the risk of developing complications. The uncertainty related to the necessity or moment of treatment initiation is based on the observation that most of the patients who do not receive treatment (with mild or active liver disease) achieve 
HBsAg seroconversion spontaneously in the first 20 years of life. $(6,7)$ Long-term research has demonstrated the fact that hepatitis B infected patients develop a similar level of antibodies to those of patients who receive Interferon treatment, but over a longer period of time. Acceleration of the seroconversion process is useful for reducing infectivity and disease activity, although HBsAg is still present in the hepatocytic core, therefore maintaining the risk of developing hepatocellular carcinoma or extra hepatic autoimmune manifestations. (8)

Currently available treatment options for chronic hepatitis B include Interferones (standard Interferon and pegylated Interferon), nucleotidic analogues (Adefovir, Tenofovir) and nucleosidic analogues (Lamivudin, Entecavir, Telbivudin, and Emtricitabin). Most of the pediatric patients remain at the immune-tolerant phase (high viral load, normal transaminases, no hepatic inflammatory activity in liver biopsy) for a long period of time. According to the current guidelines, children with a viral load above 2,000 IU $/ \mathrm{ml}$, with histologic evidence of active liver disease and transaminases higher than 1.5 times the highest normal value (two occasions over at least 6 months if $\mathrm{HBeAg}$ is positive, three occasions over the last 12 months if $\mathrm{HBeAg}$ is negative) are potential candidates for treatment initiation. Liver biopsy is controversial, but in its absence Fibromax or Fibroactitest may be used to assess inflammation and fibrosis. Currently, Interferons are considered the agents of choice, while nucleotidic/ nucleosidic analogues are used as second-line treatment. $(9,10)$

In the United States alpha Interferon is recommended for children older than 3 years of age, while Lamivudin and Entecavir may be used in children at least 2 years of age. Tenofovir and Adefovir are approved for patients 12 years or older. In the adult population, newer and more efficient molecules had been developed, therefore Interferon, Lamivudin and Adefovir are no longer the treatment of choice. Some of these are not approved in children, while others may not be used for the treatment of young patients (eg. Entecavir, Tenofovir). (11) The use of different therapies in the adult population has led to the conclusion that the best outcome is obtained when using a single agent - either pegylated alpha Interferon, Entecavir or Tenofovir. Therefore they are considered first-line treatment in naïve patients. (5)

\section{II.1 Interferons}

Standard Interferons are proteins and glycoproteins with a molecular weight that ranges from
15,000 to 27,000 Daltons. They are synthetized and secreted by the cells in response to viral infections, biological or synthetic inductors. (12) They are also involved in controlling and eliminating the viral infection from the host. Interferons are classified according to receptor specificity. Type 1 Interferons, with antiviral properties, include alpha Interferon (produced by leukocytes), beta Interferon (produced by fibroblasts) and omega Interferon. Type 2 Interferons, as for instance gamma Interferon, are the immune ones, synthetized by lymphocytes which have been activated by specific antigens or mitogenic substances. Alpha Interferon, the one used for chronic hepatitis B treatment, is a protein involved in intracellular signaling, which is mainly immunomodulatory, but also has direct but limited antiviral effect. Interferon has cytotoxic effect on viral infected cells. The main benefit of the Interferon treatment is the fact that it doesn't induce resistance and it enhances viral clearance by modulation of the host's immune system. Low viral load, high transaminases (ALT), histopathologic scores suggestive for high activity of the disease, recent infection and the absence of viral coinfection are considered positive outcome predictors of interferon treatment. The efficacy of Interferon therapy is restricted by the protein properties - low stability, short half-life (4 to 16 hours, with the highest seric value 3 to 8 hours after administration) and immunogenicity. (13-15)

Alpha 2b Interferon is recommended for chronic viral hepatitis $B$ treatment in children older than 3 years of age, with positive HBsAg for at least 6 months, signs of viral replication (positive $\mathrm{HBeAg}$, viral load higher than 2,000 IU/ml) and high ALT value. It is disputed weather liver biopsy should be performed before initiating Interferon alpha $2 \mathrm{~b}$ treatment in order to assess the severity of the liver disease. Treatment should not be given unless the disease is compensated. (12) Interferon is contraindicated in patients with decompensated liver cirrhosis (Child B or C, liver cirrhosis with hypersplenism) because even low doses can aggravate the liver disease, patients with history of hepatic encephalopathy, esophageal varices or ascites. It is not recommended for patients with high bilirubin levels (above $3 \mathrm{mg} / \mathrm{dL}$ ), low albumin (below $30 \mathrm{~g} / \mathrm{L}$ ), prolonged prothrombin time (above $3 \mathrm{sec}$ ) or hematological abnormalities (leukocytes below $4,000 / \mathrm{mm}^{3}$ or thrombocytes below $150,000 / \mathrm{mm}^{3}$ ). $(10,12,16)$ Other relative or absolute contraindications include sever renal disease, cardiovascular conditions (including coronary disease), epilepsy or other central nervous system disorders, decom- 
pensated thyroid disease (especially hyperthyroidism), autoimmune conditions (including autoimmune hepatitis), immunosuppressive treatment, severe depression, anemia or presence of autoantibodies. $(10,17)$

Most common side effects are flu-like ones (fever, myalgia, headache, etc.), but also neuropsychiatric disorders (depression, hetero and auto- aggressive behavior and suicidal conduct). Therefore close follow-up of patients during treatment is recommended. Neuropsychiatric disorders usually resolve when treatment is stopped. Another reported side effect is bone marrow aplasia, which may lead to sever cytopenia, including aplastic anemia. Interferon alpha $2 \mathrm{~b} / 2 \mathrm{a}$ may induce thyroid disorders (hypothyroidism/hyperthyroidism) but with a lower prevalence. Autoimmune side effects have been reported as well - thrombocytopenia, vasculitis, Raynaud phenomena or even rheumatoid arthritis, systemic lupus erythematosus or rhabdomyolysis. Less frequent side effects include alopecia, failure to thrive, cardiovascular disorders (hypotension, arrhythmia or tachycardia) or cerebrovascular events. (12)

The advantage of alpha Interferon is that it may be used from a young age, for a short period of time (6 to 12 months) and does not develop resistance, but it also has the disadvantage of parenteral administration and side effects.

The recommended dose is $5-10 \mathrm{MU} / \mathrm{m}^{2}$ per dose, 3 times a week for 24 weeks. If after 24/48 weeks the viral load has decreased with more than $2 \log 10$, therapy should be continued until week 48. $(6,7,18)$

Pegylated Interferon (polyethylene-glycol linked Interferon). Polyethylene glycol is a polymer used in order to modify the pharmacological characteristics of biologically active proteins, without deactivating them, lengthening the protein's duration of action by prolonging its half life, protecting it from proteolysis, and delaying its renal clearance. $(13,15)$ There are two types of pegylated alpha Interferon $-2 \mathrm{a}$ and $2 \mathrm{~b}$. Pegylated Interferon has not been approved for treatment of chronic hepatitis B in children. In adults, pegylated Interferon alpha $2 \mathrm{a}$ may be used regardless of the $\mathrm{HBeAg}$ status, while pegylated alpha $2 b$ Interferon is only approved for chronic hepatitis B in some countries. Its contraindications include autoimmune hepatitis, severe decompensated liver disease, cardiovascular disorders, HIV coinfection in patients with moderate hepatic disease, Telbivudine treatment, children younger than 3 years of age and psychiatric disorders (severe depression in particular). $(15,19,20)$

\section{II.2 Nucleosidic/nucleotidic analogues}

Nucleosidic/nucleotidic analogues suppress viral replication by discontinuing DNA synthesis process (altering the polymerase, which is essential for viral replication), but have minimal effect on DNAccc localized in the core of the hepatocyte (intrahepatic viral replication intermediate form of DNA), which is extremely stable. This makes it difficult for the virus to be eradicated and leads to the necessity of maintaining viral suppression for a long time. (15) They can be categorized into $n u$ cleotidic analogues (Adefovir and Tenofovir) and nucleosidic analogues (Lamivudin, Telbivudin and Entecavir). (15,21)

Nucleotidic/nucleosidic analogues have been considered second line therapy because of the risk of developing mutant, drug resistant hepatitis B virus strains. Resistance to oral antivirals emerges in case of prolonged treatment, reaching $23 \%$ in children after 2 years of treatment. After resistance appearance liver function tests may deteriorate showing the need of changing the therapy. A good treatment outcome can be achieved when $\mathrm{HBeAg}$ seroconversion is attained before the emergence of resistance. While resistance rate has declined, nucleotide/nucleoside analogues have been approved for adolescent use as first line treatment. For patients 12 years or older Tenofovir is the treatment of choice, while Entecavir is approved for children older than 2 years of age in the United States but only after 16 or 18 years of age in other countries. Entecavir is the best option because treatment response is good, it has few side effects and emergence of resistance is unlikely. All nucleotide/nucleoside analogues have the advantage of oral administration, as well as the disadvantage of prolonged treatment. $(6,22,23)$

Treatment compliance is one of the most important positive predictor for this class of molecules. A significant proportion of the patients who experience virological breakthrough have had a low therapeutic compliance. The dose of medication should be checked regularly because children may have a rapid weight gain rate. Treatment response at week 24 is important because patients who present complete virologic response (negative viral load) generally have a low rate of resistance. Complete response is defined by negative viral load, $\mathrm{HBeAg}$ seroconversion and normalization of transaminases. (22)

Lamivudin has few side effects, but a high rate of resistance emergence, which increases with the duration of treatment. The recommended dose is 3 $\mathrm{mg} / \mathrm{kg}$ /day (maximum $100 \mathrm{mg} /$ day), once daily. 
The success rate ranges from 25 to 35 percent. The rate of mutations is high, reaching $70 \%$ after 3 years of treatment. Long time therapy is limited by the emergence of resistance, which leads to viral replication and disease progression. Transaminases level and viral load are lower than originally when mutant strains develop, because their ability of replication is lower. Patients with Lamivudin resistance present a higher risk for associated Entecavir resistance. Stopping Lamivudin is advised if after 6 months of treatment complete suppression of viral replication is not obtained, or if there are signs of resistant mutant strains emergence. In case of development of resistance and virological breakthrou$\mathrm{gh}$, the best choice is Tenofovir or Entecavir treatment. $(5-7,18,22)$

Entecavir presents the advantage of low resistance rate even after a long period of treatment. The approval age depends upon the geographical area, ranging from 2 to 16 years. The recommended dose for naïve patients is $0.5 \mathrm{mg} /$ day, once daily. According to a research published in 2016 which evaluates efficacy and safety of Entecavir vs. placebo in the pediatric field, after 48 weeks of treatment a significant percentage of the patients have reached specific aims: viral load below $50 \mathrm{IU} / \mathrm{L}, \mathrm{HBe} A g$ seroconversion to $\mathrm{HBe} \mathrm{Ab}$, normal ALT. Although, viral suppression rates achieved in children are lower than the ones reported in adults. The reason for this is still unclear, but pediatric patients have specific immune system features, which may explain the different response to treatment. No safety issues have been reported in patients receiving Entecavir, only minor side effects, similar to those of patients in the placebo group. (6-7,18,22,23)

Tenofovir has a good response rate, does not develop resistance, has few side effects but determines decreased bone density in children and is not approved before 12 years of age. The recommended dose is $300 \mathrm{mg} /$ day for 72 weeks, with a success rate of $89 \%$. $(6,7,18,22)$

Adefovir is only approved in children 12 years or older. Its efficacy could not be proved in 2-11 age group. It is only partially efficient in patients with Lamivudine resistance and it has a high rate of resistance itself. The recommended dose is $10 \mathrm{mg} /$ day for 48 weeks. Motorization of the renal function is needed. Success rate ranges from 16 to $23 \%$. $(6,7,18,22,23)$

Duration of treatment has not been established for nucleotidic/nucleosidic analogues, but extending administration is advised 12 months after achieving negative viral load and HBeAg seroconversion. In patients who do not obtain $\mathrm{HBeAg}$ seroconversion or in $\mathrm{HBeAg}$ negative patients, long term treatment is recommended. According to adult population research, continuing treatment until HBsAg seroconversion is achieved might be safer, because serologic (HBeAg seroconversion) and virologic (negative viral load) responses are generally not sustained. As long as the treatment continues, viral load should be monitored every 3 months, a negative viral load being a positive predictor for avoiding resistance emergence. (6) Viral eradication implies eliminating DNAccc from hepatocytes, which can be achieved only by hepatocytic turnover and reactivating the host's immune system. This fact supports extending treatment 12 months after obtaining a negative viral load. In $\mathrm{HBeAg}$ negative patients increased caution is needed and treatment should not be stopped shortly after achieving negative viral load. $(6,22)$

The choice of therapy is based on positive predictors for treatment response, clinical circumstances, disease stage and potency of drugs, risk and consequences of treatment resistance. (24) Establishing first choice treatment should consider the potency of the drug (the higher the better), which should be able to rapidly reduce and maintain the viral load at undetectable levels. The ability of the molecule to control the infection in a finite period of time is another important factor. (25)

\section{ROMANIA}

Currently, in Romania, treatment criteria for chronic hepatitis B treatment are established by the Ministry of Health and National Health Insurance House, Injunction no 275/162/2015 regarding treatment prescriptions.

According to the legislation aforementioned, in order to institute specific treatment for chronic hepatitis $\mathrm{B}$ in children, following criteria should be met:

- Age 3 years or older

- At least $32.6 \mathrm{~kg}$ weight for Entecavir therapy (approved only for children older than 2 years)

- ALT value higher than two times the highest normal value

- positive HBsAg - on two occasions at least 6 months apart, regardless of $\mathrm{HBeAg}$ status

- negative IgG anti-VHD antibodies

- HBV-DNA higher than 2,000 IU/ml (26)

For patients with normal value ALT but viral load above 2,000 IU/ml, evaluation of liver fibrosis and necroinflammatory activity is needed. Evaluation should be made by Fibromax, Fibroactitest or liver biopsy. Patients with at least F1 or A1 values are eligible for antiviral treatment administration. (26) 
For Entecavir treatment initiation transaminases must be constantly elevated for at least 6 months in children with compensated liver disease and positive $\mathrm{HBeAg}$, or at least 12 months for negative $\mathrm{HBeAg}$ patients. The recommended dose is 1 tablet $(0.5 \mathrm{mg})$ per day. It should be administered until HBsAg seroconversion is obtained and 6 more months after (consolidation). Currently Entecavir tablets are the only option available in Romania, therefore it cannot be given to children weighting less than $32.6 \mathrm{~kg}$, because tablets should not be fragmented. (26)

Tenofovir, Adefovir, Lamivudin and Telbivudin are not approved for pediatric chronic viral hepatitis in our country. (26)

The recommended dose for standard interferon alpha $2 \mathrm{~b}$ is 6 million $\mathrm{U} / \mathrm{m}^{2}$ per dose $(100,000 \mathrm{IU} / \mathrm{kg}$ per dose) i.m. or s.c., 3 times a week, for 48 weeks. (26)

\section{REFERENCES}

1. World Health Organization. World Health Organization. [Online].; 2015. Available from: http://www.who.int/mediacentre/factsheets/ fs204/en/.

2. Sharma S., Carballo M., Feld J., et al. Immigration and viral hepatitis. Journal of Hepatology. 2015; 63: p. 515-522.

3. Lavanchy D., Previsani N. World Health Organization. [Online].; 2002 [cited 201601 31. Available from: http://www.who.int/csr/ disease/hepatitis/HepatitisB_whocdscsrlyo2002_2.pdf?ua=1

4. Lozano R., Naghavi M., Foreman K., et al. Global and regional mortality from 235 causes of death for 20 age groups in 1990 and 2010: a systematic analysis for the Global Burden of Disease Study 2010. Lancet. 2012; 380(9859): p. 2095-2128.

5. Hierroa L., Fischler B. Treatment of pediatric chronic viral hepatitis $B$ and C. Clinics and Research in Hepatology and Gastroenterology. 2014; 38(4): p. 415-418.

6. Sokal E., Paganelli M., Wirth S., et al. Management of chronic hepatitis B in childhood: ESPGHAN clinical practice guidelines: Consensus of an expert panel on behalf of the European Society of Pediatric Gastroenterology, Hepatology and Nutrition. Journal of Hepatology. 2013; 59(4): p. 814-829.

7. Vajro P., Veropalumbo C., Maddaluno S., et al. Treatment of children with chronic viral hepatitis: what is available and what is in store. World J Pediatr. 2013; 9(3): p. 212-220.

8. Vajro P., Veropalumbo C. Treating children with $\mathrm{HBeAg}-$ positive chronic hepatitis B: No small accomplishment. Digestive and Liver Disease. 2014; 46(12): p. 1064-1065.

9. Cholongitas E TKPC. Management of patients with hepatitis $B$ in special populations. World J Gastroenterol. 2015; 21(6): p. $1738-1748$.

10. Wang J. Antiviral therapy for hepatitis B virus-related decompensated cirrhosis. Journal of Digestive Diseases. 2012; 13(11): p. 555-557.

11. Jonas M., Lok A., McMahon B., et al. Antiviral Therapy in Management of Chronic Hepatitis B Viral Infection in Children: A Systematic Review and Meta-analysis. Hepatology. 2016; 63(1): p. 307-318.

12. Merck Sharp \& Dohme Corp. DailyMed. [Online].; 2015. Available from: http://dailymed.nlm.nih.gov/dailymed/druglnfo. cfm?setid=30789790-8317-49f9-b97b-8c5ba17b53d2.

13. Dusheiko J. Treatment of $\mathrm{HBeAg}$ positive chronic hepatitis B: interferon or nucleoside analogues. Liver International. 2012; 33(Suppl 1): p. 137-150.

\section{CONCLUSIONS}

Efficient treatment for pediatric chronic viral hepatitis is important because the evolution of the disease is longer as the moment of the infection is earlier in life, therefore the risk of developing complications, as for instance liver cirrhosis and hepatocellular carcinoma, is higher with longer evolution of the disease. Thus, early diagnosis of chronically infected patients is important and treatment should be initiated as soon as possible. The existence of more treatment options arises an opportunity for patients who are non-responsive to first line medication. New treatment options have the advantage of easy administration (oral, unlike standard Interferon which has to be given parenterally 3 times a week). The downside is the lack of compliance because of the long-term need of therapy.

14. Mihaescu G. Ebooks Universitatea Bucuresti. [Online]. Bucuresti: Universita"ttea din Bucuresti; 2003 [cited 2016 February 1. Available from: http://ebooks.unibuc.ro/biologie/mihaiescu/5b.htm\#87.

15. Wong G. Combination therapy of interferon and nucleotide/nucleoside analogues for chronic hepatitis B. Journal of Viral Hepatitis. 2014; 21(12): p. 825-834.

16. Gitlin N. Hepatitis B: diagnosis, prevention, and treatment. Clinical Chemistry. 1997; 43(8): p. 1500-1506.

17. Committee for Medicinal Products for Human Use. European Medicines Agency. [Online].; 2012. Available from: http://www.ema. europa.eu/docs/en_GB/document_library/EPAR_-_Summary_for_the public/human/000281/WC500034673.pdf.

18. Della Corte C., Nobili V., Comparcola D., et al. Management of chronic hepatitis B in children: An unresolved issue. Journal of Gastroenterology and Hepatology. 2014; 29(5): p. 912-919.

19. Committee for Medicinal Products for Human Use. European Medicines Agency. [Online].; 2014. Available from: http://www.ema. europa.eu/docs/en_GB/document_library/EPAR_-_Summary_for_the_ public/human/000395/WC500039196.pdf.

20. Merck Sharp \& Dohme Corp. DailyMed. [Online].; 2015. Available from: http://dailymed.nlm.nih.gov/dailymed/druglnfo. cfm?setid=0a8f3137-0e3a-4a60-a872-cb7d761b30e1.

21. Fung J., Lai C., Seto W., et al. Nucleoside/nucleotide analogues in the treatment of chronic hepatitis B. J Antimicrob Chemother. 2011; 66(12): p. 2715-25.

22. Chloe H., Chloe B. What physicians should know about the management of chronic hepatitis $B$ in children: East side story. World J Gastroenterol. 2014; 20(13): p. 3582-3589.

23. Jonas M., Chang M., Sokal E., et al. Randomized, controlled trial of entecavir versus placebo in children with hepatitis B envelope antigenpositive chronic hepatitis B. Hepatology. 2016; 63(2): p. 377-387.

24. Goyal S., Dixit V., Shukla S., et al. Prolonged use of tenofovir and entecavir in hepatitis B virus-related cirrhosis. Indian J Gastroenterol. 2015; 34(4): p. 286-291.

25. Coffin C., Fung S., Ma M. Management of chronic hepatitis B: Canadian Association for the Study of the Liver consensus guidelines. Can J Gastroenterol. 2012; 26(12): p. 917-938.

26. Casa Nationala de Asigurari de Sanatate. Casa Nationala de Asigurari de Sanatate. [Online].; 2015. Available from: http://www.cnas.ro/hih// theme/cnas/js/ckeditor/filemanager/userfiles/DIM/MO0172Bis_ ANEXE1_28.pdf 